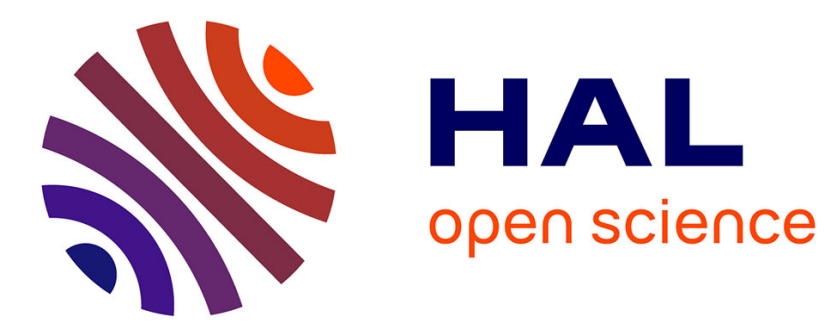

\title{
Decentralized neighborhood energy management with coordinated smart home energy sharing
}

\author{
Berk Celik, Robin Roche, David Bouquain, Abdellatif Miraoui
}

\section{To cite this version:}

Berk Celik, Robin Roche, David Bouquain, Abdellatif Miraoui. Decentralized neighborhood energy management with coordinated smart home energy sharing. IEEE Transactions on Smart Grid, 2018, 9 (6), pp.6387-6397. 10.1109/TSG.2017.2710358 . hal-02130110

\author{
HAL Id: hal-02130110 \\ https://hal.science/hal-02130110
}

Submitted on 15 May 2019

HAL is a multi-disciplinary open access archive for the deposit and dissemination of scientific research documents, whether they are published or not. The documents may come from teaching and research institutions in France or abroad, or from public or private research centers.
L'archive ouverte pluridisciplinaire HAL, est destinée au dépôt et à la diffusion de documents scientifiques de niveau recherche, publiés ou non, émanant des établissements d'enseignement et de recherche français ou étrangers, des laboratoires publics ou privés. 


\title{
Decentralized neighborhood energy management with coordinated smart home energy sharing
}

\author{
Berk Celik, Graduate Student Member, IEEE, Robin Roche, Member, IEEE, David Bouquain, and Abdellatif \\ Miraoui, Senior Member, IEEE
}

\begin{abstract}
This paper introduces a day-ahead energy management algorithm for the coordination of smart homes with renewable energy sources and energy storage systems in neighborhood areas. The aim of this study is to establish a day-ahead decentralized coordination method with appliance scheduling and energy sharing (among smart homes) to minimize the electricity bills of consumers under dynamic pricing. The energy sharing algorithm focuses on increasing the utilization of renewable sources by controlling storage units. A multi-agent system is used to model entities (smart homes, aggregator and utility) as agents and the optimization problem is solved in a decentralized manner by home agents with a genetic algorithm. The performance of the coordination algorithm is evaluated annually with and without considering forecasting errors.
\end{abstract}

Index Terms-demand response, energy management, neighborhood coordination, multi-agent systems, smart grid.

\section{NOMENCLATURE}

\section{Sets and Indexes}

$\mathbb{L}, l \quad$ Set of communication time intevals, period index

$\mathbb{T}, t \quad$ Set of actual time intervals, period index

$\mathbb{U}, u \quad$ Set of users, user index

$\mathbb{X}, x \quad$ Set of controllable appliances, appliance index

$\mathbb{Y}, y \quad$ Set of schiftable appliances, appliance index

$\mathbb{Z}, z \quad$ Set of battery control time intervals, period index

$c d \quad$ Appliance index of dish washer

$k \quad$ Iteration index

$t_{p} \quad$ Previous iteration in actual time period index

wm Appliance index of washing machine

Parameters and Constants

$\epsilon \quad$ Incident cost of last iteration

$\epsilon_{e} \quad$ Minimum cost deviation for convergence

$\kappa, \theta \quad$ Shape and scale parameter of Gamma noise

$\mu, \sigma \quad$ Mean and standard deviation of Gaussian noise

$\triangle l \quad$ Time step of communicated data

$\Delta t \quad$ Time step of actual data

$k_{\max } \quad$ Maximum iteration limit

$k_{\min } \quad$ Minimum iteration limit

$L \quad$ Communication interval

$T \quad$ Dayahead simulation length

$U \quad$ Total user number

$Z \quad$ Battery control interval

Consumption Parameters

$\epsilon_{y}^{d} \quad$ Duration length error of non-controllable appliance

$\epsilon_{y}^{s} \quad$ Start time error of non-controllable appliance

B. Celik, R. Roche, D. Bouquain, and A. Miraoui are with FEMTOST, CNRS, Univ. Bourgogne Franche-Comté, UTBM, Belfort Cedex, 90010 France e-mail: berk.celik@utbm.fr; robin.roche@utbm.fr. $d_{y}^{e} \quad$ Duration of non-controllable appliance with error

$d_{y} \quad$ Operation duration of controllable appliance

$r_{x}^{s}, r_{x}^{e} \quad$ Operation start and end time of controllable appliance

$r_{y}^{s}, r_{y}^{e} \quad$ Operation start time of non-controllable appliance

$r_{y}^{s, e} \quad$ Start time of non-controllable appliance with error

$t_{x}^{s}, t_{x}^{e} \quad$ Preferred start and end time of controllable appliance

$X_{u} \quad$ Total number of controllable appliances

$Y_{u} \quad$ Total number of non-controllable appliances

Generation Parameters

$\epsilon_{g} \quad \mathrm{PV}$ generation prediction error parameter

$G^{S T C}$ Irradiance at STC

$N_{u}^{s}, N_{u}^{p}$ Serial and parallel connected PV module numbers

$P_{u}^{p v} \quad$ Power rate of PV module at STC

Storage Parameters

$\eta_{u}^{c}, \eta_{u}^{d} \quad$ Battery charging/discharging efficiencies

$\rho_{u}^{c}, \rho_{u}^{d}$ Battery max. injected (charge/discharge) power limits

$E_{u}^{\text {bat }} \quad$ Battery capacity

$S O C_{u}^{\max }$ Maximum battery SOC

$S O C_{u}^{m i n}$ Minimum battery SOC

Variables

$\mathcal{G}(\kappa, \theta)$ Gamma distribution

$\mathcal{N}\left(\mu, \sigma^{2}\right)$ Normal distribution

$G(t) \quad$ Irradiance value

Smart Home Variables (t-domain)

$\gamma_{u}^{b}(z)$ Binary variables of battery control inputs

$\omega_{u}^{x}(t) \quad$ Binary variables of controllable appliance

$\omega_{u}^{y}(t)$ Binary variables of non-controllable appliance

$C_{u}^{*} \quad$ Electricity cost at best iteration

$C_{u}^{k} \quad$ Electricity cost at iteration k

$C_{u} \quad$ Electricity cost of smart home

$G_{u}^{e}(t)$ Irradiance value with prediction error

$P_{u}^{b}(t) \quad$ Battery charging/discharging power

$P_{u}^{c}(t) \quad$ Total consumption power of smart home

$P_{u}^{g}(t) \quad$ Generation power of PV system

$P_{u}^{I}(t) \quad$ Battery injected power

$P_{u}^{n}(t)$ Net power of smart home withou sold battery power

$P_{u}^{s}(t) \quad$ Sold battery power of smart home

$P_{u}^{x}(t)$ Consumption power of controllable appliance

$P_{u}^{y}(t)$ Consumption power of non-controllable appliance

$P_{u}^{s, d}(t)$ Decided sold battery power of smart home

$R_{u}^{n}(t) \quad$ Aggregated net power except user $u$

$R_{u}^{s}(t) \quad$ Aggregated sold battery power except user $u$

$S O C_{u}(t)$ Battery SOC value

Aggregator Variables (t-domain)

$\lambda\left(t, \mathbf{P}^{a}(t)\right)$ Neighborhood electricity price

$\mathcal{Q}\left(t, \mathbf{P}^{a}(t)\right)$ Dynamic part of electricity price

$a(t), b(t), c(t)$ Dynamic price coefficients 
$d(t) \quad$ TOU price structure

$\mathbf{P}^{a}(t) \quad$ Aggregated net power with sold power

$\mathbf{P}^{n}(t)$ Aggregated net power without sold battery power

$\mathbf{P}^{s}(t) \quad$ Aggregated sold battery power

$\mathbf{P}^{s, d}(t)$ Decided aggregated sold battery power

Smart Home Variables (l-domain)

$\hat{P}_{u}^{n}(l) \quad$ Net power of smart home

$\hat{P}_{u}^{s}(l) \quad$ Sold battery power of smart home

$\hat{R}_{u}^{n}(l) \quad$ Aggregated net power except user $u$

$\hat{R}_{u}^{s}(l) \quad$ Aggregated sold power by battery except user $u$ Aggregator Variables (l-domain)

$\hat{\lambda}\left(l, \hat{\mathbf{P}}^{a}(l)\right)$ Neighborhood electricity price

$\hat{d}(l) \quad$ TOU price structure

$\hat{\mathbf{P}}^{a}(l) \quad$ Aggregated net power with sold power

$\hat{\mathbf{P}}^{n}(l)$ Aggregated net power without sold power

$\hat{\mathbf{P}}^{s}(l) \quad$ Aggregated sold battery power

\section{INTRODUCTION}

$\mathbf{R}$ APID developments in information and communication technology (ICT) have enabled the current electricity infrastructure to become more modern and smarter, through improved sensoring, monitoring and automation capabilities. This in turn facilitates improving energy efficiency for a better utilization of resources, at all levels of the network, including the residential sector. With the implementation of advanced metering infrastructure (AMI), end-users in smart homes are changing from a passive role to an active one, by becoming active participants [1]. Smart homes are small energy systems, which may be equipped with local generation, storage and/or loads. Their inhabitants are usually modeled as self-interested players with communication and control abilities aiming to increase their social benefits (e.g., reducing their bills).

Demand response (DR) is a popular technology to control household electricity consumption, by altering and/or curtailing appliance consumption. Loads are usually shifted from onpeak to off-peak price periods and/or curtailed, sometimes with a time-varying price structure [2], [3]. DR encourages customers to reduce their load during periods of critical network congestion, when energy is more expensive, or to shift it to offpeak periods, in exchange for a rebate on their electricity bill. In this respect, smart homes have drawn significant attention by providing additional flexibility to the grid through DR programs [4]. However, DR typically only focuses on load adjustment, and neglects efficient renewable energy sources (RES) and energy storage systems (ESS) utilization. In a residential community of smart homes, adequate strategies combining DR with ESS and RES management could provide benefits for their owners, but also to service providers and utilities.

However, even if load management algorithms with RES and ESS were used in smart homes, uncoordinated decisionmaking would limit the overall performance of the proposed algorithms. For instance, unexpected issues in the distribution grid may arise, such as rebound peaks, overloading, or contingencies [5], [6], [7]. Hence a coordination mechanism is necessary so smart homes can adjust their electricity profile without negative side-effects.
Coordinated energy management in the smart grid is studied in either centralized or decentralized manners. With centralized coordination, decision-making is performed by one superior entity which can be an aggregator [8] or the utility [9]. Centralized coordination proposes effective results, however it requires detailed information about homes, which is not practical [10] and also tends to cause a heavy computation burden [11]. Moreover, users are not comfortable with the idea of seeing their appliances controlled by someone else.

On the other hand, with decentralized coordination, users are independent decision-makers who control their own electricity profile under the influence of a central entity and/or other users. In [12], a game-theoretic approach is used for the decentralized control of smart homes to reduce the peak-toaverage ratio (PAR) of an area while decreasing the electricity bill of users, with dynamic pricing. While users aim to reduce their electricity bill by scheduling their own appliances, they also enable decreasing the PAR value of the area. However, users are assumed to communicate directly with each other, which may lead to privacy issues. In [13], three decentralized algorithms are discussed and use a game theoretic approach: i) with a central entity, ii) without a central entity with synchronous decision-making iii) without central entity with asynchronous decision-making. In the first method, users communicate with the central unit to gather the aggregated profile. In the synchronous method, users update their profiles simultaneously, while they use a different step size in the asynchronous model. In all models, dynamic pricing is used to reduce the PAR of the area and users' bills.

In [14], the decentralized control problem is solved using a Vickrey-Clarke-Groves mechanism. The properties of the proposed method are compared with two other methods: $i$ ) a competitive equilibrium problem where users are fixed price takers, and ii) a game of Nash equilibrium problem where users are price anticipative, and the effect of other users consumption is considered in the price. In the proposed model, users take into account the utility function of others to represent the level of user satisfaction while trying to maximize their own benefit. By this way, the presented method proposes better results compared to the other cases. However, this study requires detailed data sharing between users, which also leads to privacy concerns.

Other studies additionally consider RES and ESS in smart homes. In [15], a decentralized online algorithm which takes into account the uncertainty on renewable generation, consumption and cost is modeled to reduce the electricity cost of smart homes. However, the algorithm does not consider two-way electricity transfers between smart homes and the grid. Thus the study only focuses on better utilizing home generation with self-consumption. In [16], an agent-based electricity trading algorithm with residential RES and ESS is studied. For home decision-making, home agents decide to buy/sell electricity to the grid, charge/discharge the battery or ignore the low priority loads to maximize their outcome. To influence the home agents, two price rates are defined: one for buying and one for selling energy to the grid. However, although the battery is used and energy trading is established, only instant residual RES generation is used for trading and 
the ESS is deployed only for self-consumption.

Lastly, in our previous study [17], we introduced a coordinated energy management algorithm using time-of-use (TOU) pricing and a feed-in-tariff (FIT) with a constant incentive for increasing self-consumption in the neighborhood. By using TOU, FIT and the incentive, the final area electricity price was lower than the grid price, which favored energy trading inside the neighborhood. With this algorithm, home batteries were also able to provide energy for neighborhood consumption. However the effect of the aggregated profile on the area electricity price was not studied. Moreover, the algorithm relied on an externally-funded incentive and FIT.

Although these studies proposed various coordination mechanisms with a focus on different aspects, they do not consider energy sharing through RES generation and battery use in a decentralized manner. In this paper, we introduce a decentralized coordination method to reduce the electricity bills of the users by increasing local renewable energy utilization in the neighborhood with a dynamic price model. We consider a scenario where RES and ESS are owned by the users and renewable generation is shared among neighbors, instead of being fed back to the grid. By this way, users can help decrease carbon emissions, as well as their electricity consumption costs, and PAR of the area can also be decreased. Two decentralized coordination models are presented, group-based and turn-based. Both rely on an architecture based on the multi-agent systems (MAS) concept, which enables dynamic interactions among competitive and cooperative entities to achieve predefined objectives [18]. The predefined program (optimization problem) is formulated and solved by all home agents using the same genetic algorithm (GA). In the literature, various methods are used to solve similar optimization problems, such as linear programming [19], approximate dynamic programming [20], mixed integer nonlinear programming [21] and also other heuristic methods such as particle swarm optimization [22]. However, we prefer to use GA for solving this optimization problem due to the ability to find nearoptimal solutions within acceptable computation times, as well as the flexibility of the algorithm to a wide variety of problems.

The key contributions of this paper are summarized as follows:

1) The impact of sequence (group-based vs. turn-based) on coordination is analyzed, by comparing the performance of two decentralized approaches (group-based and turnbased ) with annual simulations. All smart homes (with and without RES and/or ESS) achieve savings, as does the utility in terms of lower peak demand.

2) In addition to costs and PAR, three different metrics (SR-01 to SR-03) are introduced. The algorithms provide better results compared to a base case, even when considering uncertainty on consumption and renewable generation.

3) Three data profile time resolutions are used the optimization problem to enable detailed modeling, reduce the computational burden, or ensure the privacy of the users.

4) The proportional source matching method is created to eliminate mismatches occurring in real-time, after the day-ahead optimization.
The rest of the paper is organized as follows: the system model is presented in Section II and the problem formulation is given in Section III. The developed coordination mechanisms are described in Section IV. Results are given in Section V and discussed in Section VI. The paper is concluded in Section VII.

\section{SySTEM MODEL}

We consider an electricity network with one utility company connected to a set of smart homes by an AMI and through an aggregator. The aggregator is a third-party entity that is able to provide ancillary services to the utility, and can play a role in coordinating smart homes for better energy management [23]. In this study, the aggregator receives electricity profiles data (i.e., the home net power profile $P_{u}^{n}(t)$ and the home sold battery power profile $P_{u}^{s}(t)$, described in Section III) from the smart homes, determines the aggregated neighborhood profile and dynamic price, and then sends them back to the smart homes.

\section{A. Home Energy System}

In the following, we consider smart homes which can consume, generate and/or store electric energy while being connected to the grid. Through AMI, smart homes communicate with other entities and control their own electricity profile. In this study, we assume that each user is equipped with a smart meter and a controller, and only communicates with the aggregator (and not with other users) due to privacy concerns.

Depending on their controllability, 13 types of appliances are modeled in two groups: controllable and non-controllable appliances. In this study, the washing machine, the clothes dryer and the dish washer are considered controllable, while others (TV, computer, coffee maker, etc.) are assumed to be non-controllable appliances. While other loads may be controlled, these three are the most commonly used in the literature for creating realistic control scenarios [24]. The operation of appliances is defined by binary variables $\omega_{u, t} \in\{0,1\}$ at $\forall t \in \mathbb{T}$ ( 0 for off, 1 for running) as:

$$
\begin{array}{ll}
\omega_{u}^{x}(t)=\left\{\begin{array}{ll}
1 & : t \in\left[r_{x}^{s}, r_{x}^{e}\right] \\
0 & : t \in \mathbb{T}-\left[r_{x}^{s}, r_{x}^{e}\right]
\end{array}\right\}, & \forall x \in \mathbb{X} \\
\omega_{u}^{y}(t)=\left\{\begin{array}{ll}
1 & : t \in\left[r_{y}^{s}, r_{y}^{e}\right] \\
0 & : t \in \mathbb{T}-\left[r_{y}^{s}, r_{y}^{e}\right]
\end{array}\right\}, \quad \forall y \in \mathbb{Y}
\end{array}
$$

We assume that the consumption power of the appliances is fixed during the working period of appliances, thus the smart home consumption is formulated by:

$$
P_{u}^{c}(t)=\sum_{x=1}^{X_{u}} P_{u}^{x}(t) \cdot \omega_{u}^{x}(t)+\sum_{y=1}^{Y_{u}} P_{u}^{y}(t) \cdot \omega_{u}^{y}(t)
$$

In this study, to increase diversity among the consumption profiles of smart homes, loads are modeled according to a probabilistic model. According to the consumption rates shown in Table I, the number of electricity appliances is determined probabilistically for each smart home. For example, a smart home with a high energy consumption is more likely to be equipped with more than one TV than a low-rated smart 
TABLE I

Penetration LeVel of Home Resources and PV module PARAMETER.

\begin{tabular}{lccccc}
\hline Consumption Rate & $\mathrm{PV}$ & $\mathrm{N}_{u}^{s}$ & $\mathrm{~N}_{u}^{p}$ & $\mathrm{P}_{u}^{p v}$ & Battery \\
\hline Very Low (10\%) & $30 \%$ & $1-4$ & $1-4$ & $160-200$ & $20 \%$ \\
Low (30\%) & $40 \%$ & $2-5$ & $1-4$ & $160-200$ & $30 \%$ \\
Medium (25\%) & $50 \%$ & $2-5$ & $2-5$ & $180-200$ & $40 \%$ \\
High (25\%) & $60 \%$ & $3-6$ & $2-5$ & $220-240$ & $50 \%$ \\
Very High (10\%) & $70 \%$ & $3-6$ & $3-6$ & $220-240$ & $60 \%$ \\
\hline
\end{tabular}

home. Parameter values in Tables I are arbitrarily defined to reflect a realistic case.

Additionally, end-users have only one from each type of controllable appliance. The number of appliances, power rates, and operation duration are given in Table II. For realistic cases, we assume that some appliances can be used more than once ('Multiple'), such as lights. When an appliance is turned on, it is used at the minimum for the duration given in Table II. After that, it can be used for at least the same duration, or be turned off.

Photovoltaic (PV) systems are considered as the only RES in smart homes. The power produced by a PV array is formulated as:

$$
P_{u}^{g}(t)=N_{u}^{s} \cdot N_{u}^{p} \cdot P_{u}^{p v} \cdot\left(G(t) / G_{S T C}\right)
$$

The number of modules and their rated power are also determined probabilistically. The corresponding probabilities are given in Table I. We assume that smart homes with a high consumption rate are more willing to install PV with a high rated power.

To store the surplus energy generated by PV panels in the smart homes, a battery is used as an ESS. Battery power is impacted by charging $\left(P_{u}^{b}(t)>0\right)$ and discharging $\left(P_{u}^{b}(t) \leq\right.$ 0 ) efficiencies and operated between minimum and maximum state-of-charge (SOC) levels.

$$
\begin{gathered}
P_{u}^{b}(t)=\left\{\begin{array}{ll}
P_{u}^{I}(t) \cdot \eta_{u}^{c} & : P_{u}^{I}(t)>0 \\
P_{u}^{I}(t) / \eta_{u}^{d} & : P_{u}^{I}(t) \leq 0
\end{array}\right\} \\
\rho_{u}^{d} / \eta_{u}^{d} \leq P_{u}^{b}(t) \leq \rho_{u}^{c} \cdot \eta_{u}^{c}
\end{gathered}
$$

TABLE II

SMART Home APPliance Data.

\begin{tabular}{lccc}
\hline Appliance & Amount & Power (W) & Duration (min.) \\
\hline Lights & $2-8$ & 25 & $15-$ Multiple \\
Kettle & $0-1$ & 450 & $15-$ Multiple \\
Microwave & $0-1$ & 800 & $10-$ Multiple \\
Vacuum Cleaner & $0-1$ & 700 & $30-$ Single \\
Television & $0-3$ & 150 & $30-$ Multiple \\
Computer & $1-5$ & 250 & $30-$ Multiple \\
Iron & $0-1$ & 650 & $30-$ Single \\
Hair Dryer & $0-1$ & 200 & $10-$ Multiple \\
Coffee Maker & $0-1$ & 300 & $10-$ Multiple \\
Toaster & $0-1$ & 500 & $10-$ Multiple \\
Washing Machine & $0-1$ & 800 & $90-$ Single \\
Clothes Dryer & $0-1$ & 1000 & $90-$ Single \\
Dish Washer & $0-1$ & 850 & $60-$ Single \\
\hline
\end{tabular}

$$
\begin{gathered}
S O C_{u}(t)=S O C_{u}(t-1)+\left(P_{u}^{b}(t) \cdot \Delta t\right) / E_{u}^{b a t} \\
S O C_{u}^{\min } \leq S O C_{u}(t) \leq S O C_{u}^{\max }
\end{gathered}
$$

As for PV system modeling, the ownership of the battery system in the smart homes is determined probabilistically, and according to the probabilities given in Table I. The battery capacity is related to the installed PV installation capacity.

\section{B. Forecasting Error Model}

Forecasting errors are considered for non-controllable appliances loads and PV generation. Gaussian distributions are used to model the start time and duration errors of appliance operation:

$$
\begin{aligned}
r_{y}^{s, e} & =r_{y}^{s}+\epsilon_{y}^{s} \\
d_{y}^{e} & =d_{y}+\epsilon_{y}^{d}
\end{aligned}
$$

Errors are determined by a Gaussian distribution $\mathcal{N}\left(\mu, \sigma^{2}\right)$ [25]. The mean $\mu$ is chosen equal to 0 , but the standard deviation $\sigma$ is assumed variable in terms of minutes according to the quantity and appliance type.

In this study, we assume that all home agents are forecasting their generation profiles individually. Prediction errors are thus different in each smart home, even though they are located in the same geographical region. To perform forecasting of irradiance value, the Gamma distribution [26] is used:

$$
G_{u}^{e}(t)=G(t) \times \epsilon_{g}
$$

Errors for generation are determined using gamma noise with distribution $\mathcal{G}(\kappa, \theta)$, where $\kappa=210$ is the shape parameter, and $\theta=0.005$ is the scale parameter.

\section{Electricity Cost}

This section describes the dynamic pricing scheme used for billing consumers in the neighborhood. The price is determined based on two variables: $i$ ) a base price at the upper level of the neighborhood, and ii) the aggregated consumption fluctuation in the neighborhood. We assume that the utility, at the upper level, determines the base price for each time slot (typically hourly). Then, the aggregator agent determines the dynamic price associated to the base price and the aggregated consumption of the neighborhood. To model the dynamic part, a quadratic function is used [27] where the unit price is related to the aggregated profile:

$$
\mathcal{Q}\left(t, \mathbf{P}^{a}(t)\right)=a(t) \cdot\left|\mathbf{P}^{a}(t)\right|^{2}+b(t) \cdot\left|\mathbf{P}^{a}(t)\right|+c(t)
$$

with $a(t)>0$, and $b(t), c(t) \geq 0$. We consider the base structure to be equal to the French regulated TOU tariff [17], and merge it with (9):

$$
\lambda\left(t, \mathbf{P}^{\mathbf{a}}(t)\right)=\left\{\begin{array}{ll}
d(t)-\mathcal{Q}\left(t, \mathbf{P}^{\mathbf{a}}(t)\right) & : \mathbf{P}^{\mathbf{a}}(t) \leq 0 \\
d(t)+\mathcal{Q}\left(t, \mathbf{P}^{\mathbf{a}}(t)\right) & : \mathbf{P}^{\mathbf{a}}(t)>0
\end{array}\right\}
$$

In this model, the central entity enables users to control resources not only according to the aggregated profile, but also according to the base structure due to the connection to the main grid. Moreover, the presented price model is used as an FIT, which enables reverse power flow when smart home generation is higher than consumption. 


\section{PROBlem Formulation}

In this section, the decentralized optimization problem is formulated to show how home agents optimize their social benefits in terms of cost reduction by scheduling their appliances and controlling ESS. The proposed ESS control algorithm addresses the energy sharing problem of the smart home in the neighborhood. Firstly, we assume that users define a control interval for the scheduling of controllable appliances. In this interval, home agents pick the most beneficial time for running appliances during low price hours without reducing user comfort [28]. The constraint is formulated by:

$$
\left[r_{x}^{s}, r_{x}^{e}\right] \in\left[t_{x}^{s}, t_{x}^{e}\right]
$$

Secondly, a clothes dryer should logically run after the washing machine has finished [29]. The operation time of a clothes dryer should be thus controlled by taking into account the washing machine operation time as:

$$
\begin{gathered}
r_{w m}^{s}<r_{c d}^{s}-\left(r_{w m}^{e}-r_{w m}^{s}\right) \\
t_{w m}^{s}<t_{c d}^{s}-\left(r_{w m}^{e}-r_{w m}^{s}\right)
\end{gathered}
$$

Lastly, the battery power is determined for the smart homes that have one. Home agents can discharge a battery to use and/or sell energy during on-peak hours to increase their benefit, and reduce the aggregated peak load of the neighborhood. It should be noted that, in this work, batteries are only allowed to discharge for selling energy to the neighborhood (and not to the main grid). Home agents use the aggregated data $\left(\mathbf{P}^{n}(t)\right.$ and $\left.\mathbf{P}^{s}(t)\right)$ with the dynamic price $\left(\lambda\left(t, \mathbf{P}^{a}(t)\right)\right)$ received from the aggregator. After home agents receive it, they subtract their previous electricity profiles from the aggregated profile:

$$
\begin{aligned}
R_{u}^{n}(t) & =\mathbf{P}^{n}(t)-P_{u}^{n}\left(t_{p}\right) \\
R_{u}^{s}(t) & =\mathbf{P}^{s}(t)-P_{u}^{s}\left(t_{p}\right)
\end{aligned}
$$

Then home agents determine $P_{u}^{b}(t)$ using $R_{u}^{n}(t)$ and $R_{u}^{s}(t)$ with (15). However, controlling the battery for each time interval ( $T$ inputs) for the day-ahead optimization (for example 1440 for 1-min. modeling) would cause a significant computation burden. Therefore, a battery control set $\mathbb{Z}$ is defined to decrease the number of inputs from $T$ to $T / Z$, and speed-up the algorithm. For the control of the battery, binary variables $\gamma_{u}^{b}(z) \in\{0,1\}$ are defined for logically controlling the battery charging/discharging operations. $P_{u}^{b}(t)$ is determined by:

$$
\begin{aligned}
& P_{u}^{b}(t)= \\
& \left\{\begin{array}{ll}
\text { fullcharge } & : \gamma_{u}^{b}(z)=0, P_{u}^{g}(t)>0 \\
\text { idle } & : \gamma_{u}^{b}(z)=0, P_{u}^{g}(t) \leq 0 \\
\text { charge } & : \gamma_{u}^{b}(z)=1, P_{u}^{g}(t) \geq P_{u}^{c}(t)+R_{u}^{n}(t)-R_{u}^{s}(t) \\
\text { discharge } & : \gamma_{u}^{b}(z)=1, P_{u}^{g}(t)<P_{u}^{c}(t)+R_{u}^{n}(t)-R_{u}^{s}(t)
\end{array}\right\}
\end{aligned}
$$

where fullcharge is charging without using RES generation for consumption $\left(P_{u}^{b}(t)=P_{u}^{g}(t) \cdot \eta_{u}^{c}\right)$, idle is waiting $\left(P_{u}^{b}(t)=0\right)$, charge is first using generation for consumption then charging with the surplus generation $\left(P_{u}^{b}(t)=\left(P_{u}^{g}(t)-\left(P_{u}^{c}(t)+R_{u}^{n}(t)-R_{u}^{s}(t)\right)\right)\right.$. $\left.\eta_{u}^{c}\right)$, and discharge is discharging $\left(P_{u}^{b}(t)=\right.$ $\left.-\left(P_{u}^{c}(t)-P_{u}^{g}(t)+R_{u}^{n}(t)-R_{u}^{s}(t)\right) / \eta_{u}^{d}\right)$. According to

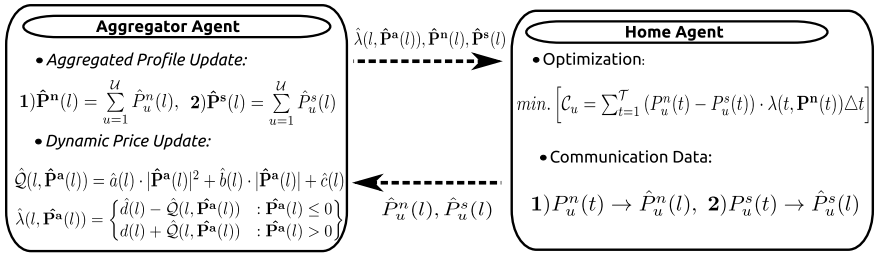

Fig. 1. Coordination diagram of agents.

(15), the battery only discharges when $\gamma_{u}^{b}(z)=1$, hence the sold battery power is calculated with:

$$
P_{u}^{s}(t)=P_{u}^{b}(t)-P_{u}^{c}(t)
$$

By applying the above comparisons, the sold power $P_{u}^{s}(t) \geq$ 0 by battery discharge $\left(P_{u}^{b}(t) \leq 0\right)$ is calculated. According to (15)-(16), if there are home and neighborhood consumption at the same time when battery discharging is decided, the discharged power basically is first used for home consumption and then sold to the neighbors.

After that, home agents determine the net electricity profile of their smart home with:

$$
P_{u}^{n}(t)=P_{u}^{c}(t)-P_{u}^{g}(t)+P_{u}^{b}(t)+P_{u}^{s}(t)
$$

and optimize the following objective function with the set of constraints to minimize the daily electricity bill of the users.

$$
\begin{gathered}
\min \left[\mathcal{C}_{u}=\sum_{t=1}^{T}\left(P_{u}^{n}(t)-P_{u}^{s}(t)\right) \cdot \lambda\left(t, \mathbf{P}^{a}(t)\right) \cdot \Delta t\right] \\
\text { s.t. } \quad(1),(2),(5),(6),(11),(12),(13)
\end{gathered}
$$

This optimization problem is solved by all home agents using GA. Note that $P_{u}^{s}(t)$ is removed in (17) and added in (18). They are needed separately during the data exchange described in Section IV. Two time resolutions are used for solving the optimization problem for two reasons: first, to use and determine the electricity profiles with a high resolution with " $t$ ", and second, to reduce the computation time by reducing the number of input numbers of the day-ahead optimization problem with a lower resolution " $z$ ".

\section{COORDINATION With EnERgy SHARING}

In this study, we propose two coordination models for the neighborhood area: group-based and turn-based. In both models, the same optimization problem is solved and the same communication principle is used as in Fig. 1, but the coordination architecture is different.

Before presenting the algorithms, we assume that: $i$ ) home agents do not communicate with each other, ii) the calculated average data is used to reduce the communication requirements with communication time set $\mathbb{L}$. In this respect, when a message is sent, data size is modified as $[1 \times T] \rightarrow[1 \times T / L]$ and reconverted as $[1 \times T / L] \rightarrow[1 \times T]$ when a message is received [11]. 


\section{A. Group-Based Coordination Model}

In this section, the group-based model is described in Algorithm 1. Firstly, the utility agent sends $\hat{d}(l)$ to the aggregator agent at the beginning of the day. As the aggregator agent does not have any information about the home profiles at $k=1$, it sends $\hat{d}(l), \hat{\mathbf{P}}^{\mathbf{n}}(l)=0$ and $\hat{\mathbf{P}}^{\mathbf{s}}(l)=0$ to the home agents. Home agents receive this data and run the optimization with (18), all at the same time. After that, they send the home net power profile $\hat{P}_{u}^{n}(l)$ and the home sold power profile by battery discharge $\hat{P}_{u}^{s}(l)$ to the aggregator, which determines the aggregated profile with the dynamic price and sends them back to the home agents. This process continues until convergence is reached (see Section IV-C).

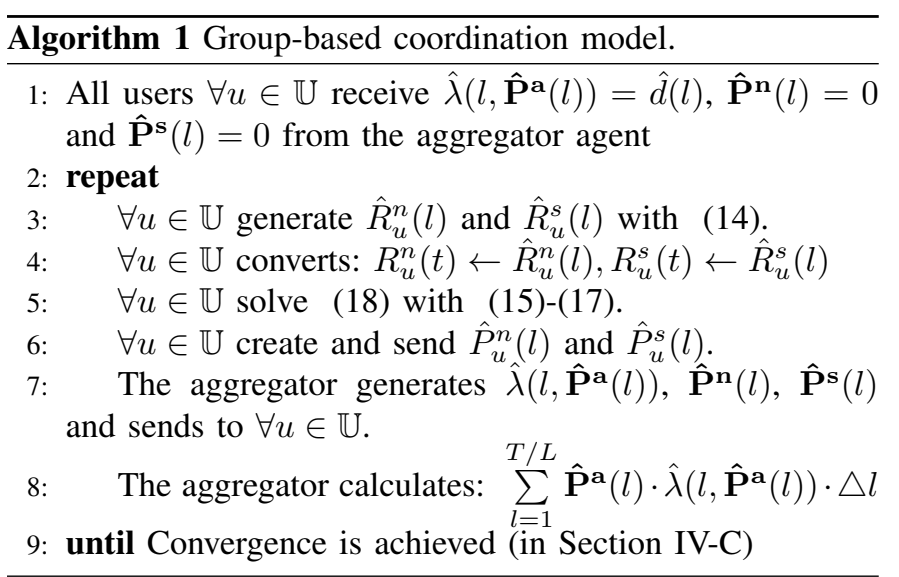

As all agents run the optimization simultaneously with Algorithm 1, $\mathbf{P}^{\mathbf{s}}(t)$ may become higher than the aggregated consumption $\mathbf{P}^{\mathbf{n}}(t)$ (which can lead to mismatches) during the procedure in two times: $i$ ) at the end of each iteration, and $\mathrm{ii}$ ) when final decisions are converted from the $l$-domain to the $t$-domain. Therefore, for the first case, the aggregator agent determines the price by comparing $\hat{\mathbf{P}}^{\mathbf{s}}(l)$ and $\hat{\mathbf{P}}^{\mathbf{n}}(l)$ in (19). There is a possibility that home agents can discharge their battery for neighborhood consumption at the same time due to simultaneous optimization, which may lead to having $\hat{\mathbf{P}}^{\mathbf{n}}(l) \leq \hat{\mathbf{P}}^{\mathbf{s}}(l)$ when $\hat{\mathbf{P}}^{\mathbf{n}}(l)>0$.

$$
\hat{\mathbf{P}}^{\mathbf{a}}(l)=\left\{\begin{array}{ll}
0 & : \hat{\mathbf{P}}^{\mathbf{n}}(l)>0, \hat{\mathbf{P}}^{\mathbf{n}}(l) \leq \hat{\mathbf{P}}^{\mathbf{s}}(l) \\
\hat{\mathbf{P}}^{\mathbf{n}}(l)-\hat{\mathbf{P}}^{\mathbf{s}}(l) & : \hat{\mathbf{P}}^{\mathbf{n}}(l)>0, \hat{\mathbf{P}}^{\mathbf{n}}(l)>\hat{\mathbf{P}}^{\mathbf{s}}(l) \\
\hat{\mathbf{P}}^{\mathbf{n}}(l) & : \hat{\mathbf{P}}^{\mathbf{n}}(l) \leq 0
\end{array}\right\}
$$

For the second case, a method called proportional source matching (PSM) is applied, where the sold power of home agents is proportionally determined based according to total decided sold power after the convergence is reached. If there is one seller $\left(\mathbf{P}^{\mathbf{s}}(t)=P_{u}^{s}(t)\right)$ and the sold battery discharge power is less than or equal to the aggregated consumption $\left(\mathbf{P}^{\mathbf{n}}(t) \geq P_{u}^{s}(t)\right)$, no extra calculation is required $\left(P_{u}^{s}(t)=\right.$ $\left.P_{u}^{s, d}(t)\right)$. Otherwise, when the number of sellers is higher than one $\mathbf{P}^{\mathbf{s}}(t)>P_{u}^{s}(t)$ and $\mathbf{P}^{\mathbf{s}}(t)>\mathbf{P}^{\mathbf{n}}(t)$, then:

$$
P_{u}^{s}(t)=\mathbf{P}^{\mathbf{n}}(t) \cdot\left(P_{u}^{s, d}(t) / \mathbf{P}^{\mathbf{s}, \mathbf{d}}(t)\right)
$$

If a mismatch occurs between the $l$-domain and the $t$ domain, the aggregator stabilizes the system by determining $P_{u}^{s}(t)$ for each user based on the ratio between $P_{u}^{s, d}(t)$ and $\left.\mathbf{P}^{\mathbf{s}, \mathbf{d}}(t)\right)$.

\section{B. Turn-Based Coordination Model}

In the turn-based model, as for the group-based model, home agents communicate the average calculated data and do not exchange messages with each other. Also, the aggregator agent applies PSM with (20) to cover mismatches between communication and actual data after the final iteration. A difference is that home agents communicate and solve the optimization problem one after another. Hence they do not need to apply (19) because they are informed of changes after each home optimization $\left(\hat{\mathbf{P}}^{\mathbf{a}}(l)=\hat{\mathbf{P}}^{\mathbf{n}}(l)-\hat{\mathbf{P}}^{\mathbf{s}}(l)\right)$. The procedure is described in Algorithm 2.

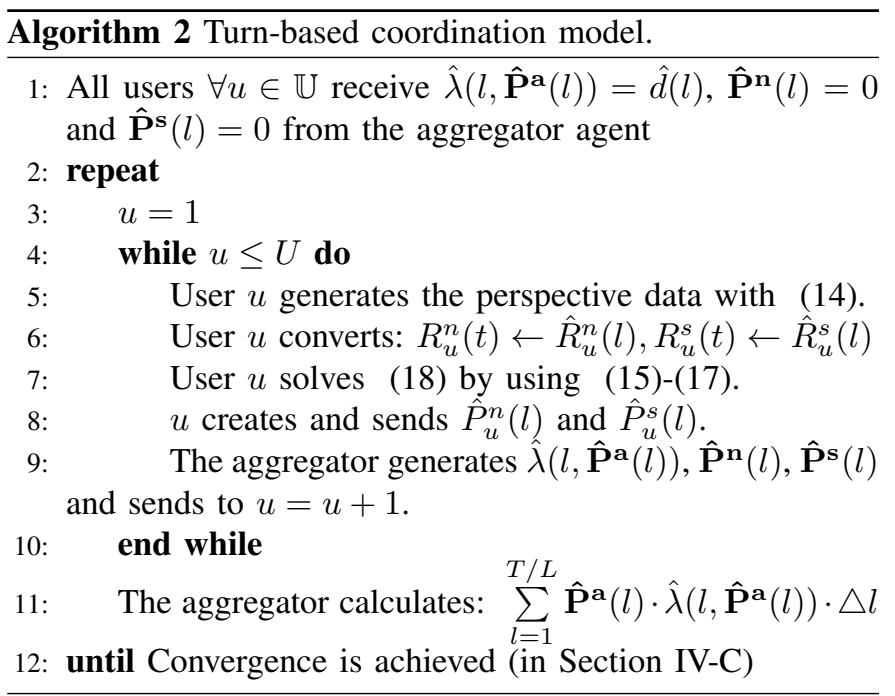

In this model, $k$ is increased after the $U$-th home agent optimization. Then, if convergence is not achieved, each user $u \in \mathbb{U}$ runs the optimization again.

\section{Convergence}

For the proposed coordination models, algorithm convergence is determined as follows. The system reaches convergence if the total cost does not fluctuate by more than $\epsilon_{e}$. Otherwise, iterations continue until the allowed maximum number iteration is reached. Then, the aggregator agent ends the process and considers the latest decision of smart homes at $k_{\max }$ as the final.

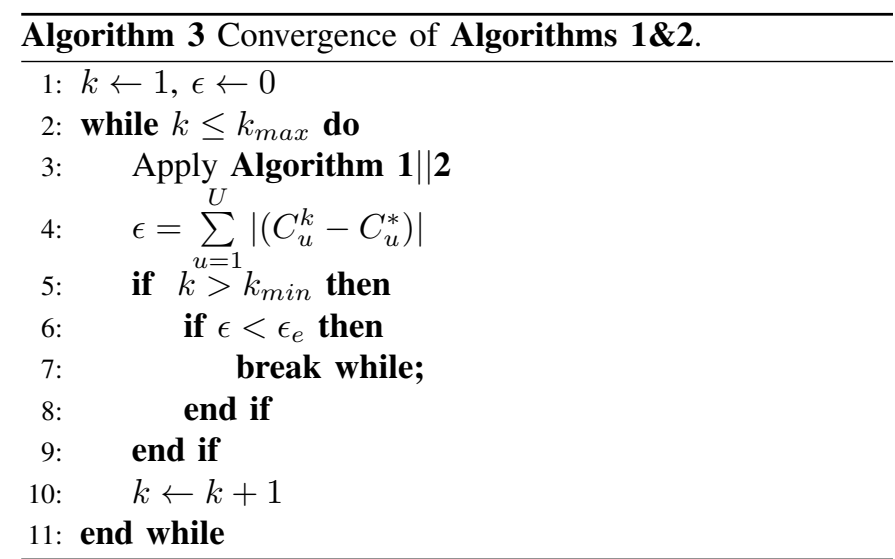



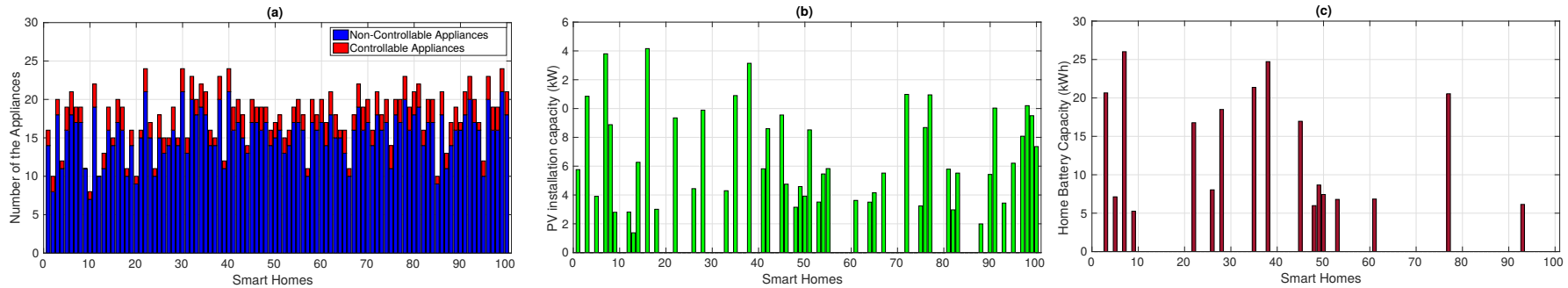

Fig. 2. Simulation setup. (a) Controllable and non-controllable appliance numbers, (b) PV capacities, (c) battery capacities in smart homes.

\section{Simulation Results}

In this study, we use one utility company, one aggregator and $U=100$ smart homes. The number of smart appliances and the installed capacities of PV and battery systems in smart homes are shown in Fig. 2. The number of PV (48) and battery (17) are determined probabilistically. An example smart home profile is given in Fig. 3. The probabilistic model described in Section II-A is used to determine appliances consumption and PV generation in smart homes. Solar radiation data is taken from a weather station located in Belfort, France.

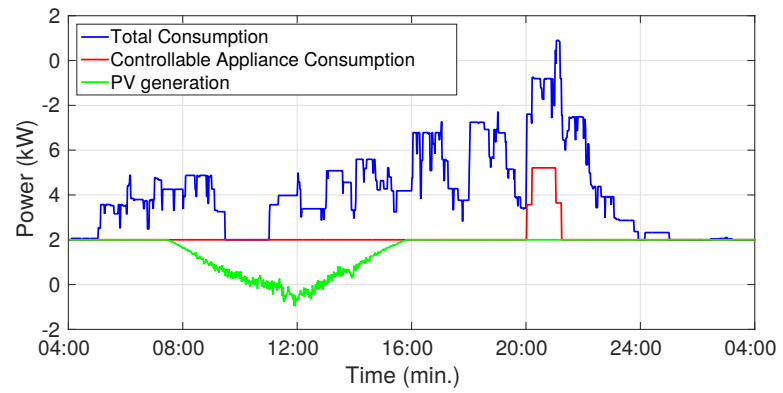

Fig. 3. Smart home total and controllable appliance consumption profiles with PV generation.

The controllable appliance consumption and the PV generation ratio with respect to the total home consumption vary between $4-7 \%$ and $5-89 \%$, respectively. The cause of these small ratios in the controllable appliance consumption share is that these appliances are not working every day in a year. When it is used, a controllable appliance working probability is decreased for the next day, and vice versa if it is not used. In this study, 229 controllable appliances are available in the neighborhood and used 38,349 times in a year (less than $229 \times 365=83585$ ). Furthermore, we assume that controllable appliances can only be scheduled until $1 \mathrm{am}$, because of the end-users noise preferences.

Lastly, to perform annual simulations, a rolling-horizon process is included with the optimization algorithm, as in [17]. Home agents thus solve the optimization problem for two days $(T=2880)$, and repeat it every day with the first day result at $t=1440$ as the initial step (for the battery SOC) of the next simulation roll. For communication, home agents send the average calculated data for each $L=30$ minutes. Smart homes with a battery use a battery control interval of $Z=30$ minutes. The same co-simulation platform (MATLAB/JADE) as in [11] is used.
In the following sections, the performance of the proposed algorithms is compared with two scenarios: Baseline and Selfish Control. In Baseline, smart homes do not communicate with the aggregator, share energy nor control their appliances. They are modeled as passive users. In Selfish Control, homes can control their electricity appliances and batteries according to TOU without coordination and energy sharing.

\section{A. Daily Analysis without Forecasting Errors}

We first run simulations without considering forecasting errors, on an arbitrarily chosen day (the 153th day) of the year. Results are summarized in Fig. 4. In Fig. 4(a), the coordination methods show better performance compared to the Baseline and Selfish Control methods, both in terms of costs and peak power demand. Algorithm 2 shows a slightly better performance, and enables saving $1.65 €$ more compared to Algorithm 1. Selfish Control seems less effective, because users are not aware of the aggregated consumption profile, thus they have the possibility to start their controllable appliances at the same time, which causes a $2.5 \%$ peak and a $16.8 \%$ cost increase in the neighborhood. On the other hand, there is a significant peak (14\%) and cost (20\%) reduction with both Algorithms 1\&2 compared to other methods. As it updates electricity profiles and price after each home agent optimization, Algorithm 2 shows slightly better results (3.46\% peak, $0.7 \%$ cost) than Algorithm 1.

Secondly, energy comparisons are shown in Fig. 4(b). Even though there is no coordination for Baseline and Selfish Control, there is shared energy during high PV generation hours which occurs naturally (physically, if there is enough load, surplus energy during daylight is used locally inside the neighborhood instead of being fed back to the main grid). Also, self-consumption is higher in these two algorithms as sharing energy by battery discharge is not allowed. Therefore, batteries are just discharged for the own consumption of the smart homes. On the other hand, home agents increase energy sharing and decrease self-consumption by discharging batteries for the neighborhood with both Algorithms 1\&2. These algorithms achieve decreasing the energy purchased from the utility. As there is no coordination, home agents discharge their batteries just for their own consumption, so there is always more energy left in the batteries, as shown in Fig. 4(c). On the other hand, with this coordination algorithm, home agents are able to discharge their batteries for neighborhood consumption and decrease the energy purchased from the utility. 

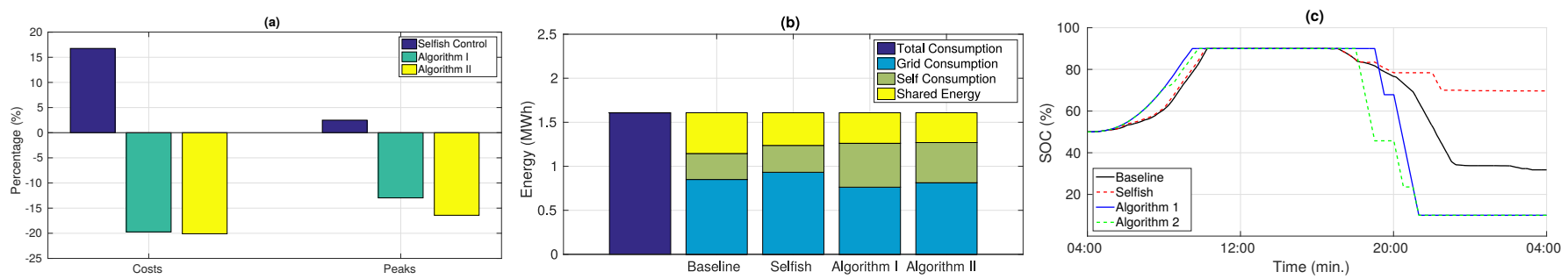

Fig. 4. One day simulation results. (a) Neighborhood cost and peak consumption, (b) energy comparison, (c) SOC profile of a smart home with a battery.

TABLE III

DAILY ELECTRIC ENERGY MANAGEMENT WITH (WE) AND WITHOUT (WOE) FORECASTING ERRORS.

\begin{tabular}{clllllllll}
\hline & \multicolumn{2}{l}{ Cost $(€)$} & \multicolumn{2}{c}{ Cost $(\%)$} & \multicolumn{2}{c}{ Peak (kW) } & \multicolumn{2}{c}{ Peak (\%) } \\
\cline { 2 - 8 } & WoE & WE & WoE & WE & WoE & WE & WoE & WE \\
\hline Baseline & 94 & - & - & - & 192 & - & - & - \\
Selfish & 109 & 110 & -16.8 & -17.5 & 197 & 206 & -2.5 & -7.6 \\
Algorithm 1 & 75 & 78 & 20.1 & 16.6 & 167 & 171 & 13.0 & 10.8 \\
Algorithm 2 & 74 & 77 & 20.8 & 18.3 & 160 & 163 & 16.4 & 15.2 \\
\hline
\end{tabular}

However, for Baseline and Selfish Control, the battery energy saved at the end of the day can still be used for next day consumption, which introduces a bias in results comparison. On the contrary, batteries can be fully discharged with Algorithms 1\&2 by providing energy to the neighborhood for increasing self-benefit. To account for this fact, we perform an annual simulation in Section V-C.

\section{B. Daily Analysis with Forecasting Errors}

We now consider the same day, but consider forecasting errors. The percentage of prediction errors are calculated by the symmetrical absolute percentage errors [30] for the aggregated consumption $(13.84 \%)$ and generation profiles $(9.52 \%)$ of the neighborhood. The cost and peak consumption results for both with/without considering forecasting errors are given in Table III, with absolute and percentage values. Percentages are calculated with respect to Baseline results.

As expected, forecasting errors negatively impact all algorithms for both cost and peak reduction efficiencies. However, numerical results show that the proposed algorithms still provide better performance compared to Baseline due to their coordination and energy sharing ability.

\section{Annual Results}

In this section, annual results are determined for both with/without considering forecasting errors. In Fig. 5, the neighborhood cost and peak consumption without considering forecasting errors are given for a year. For all algorithms, the total peak and cost results exhibit differences due to changes in PV generation during seasons. Although there is a slight difference in the cost results, the proposed Algorithms 1\&2 provide more benefits in lowering peak consumption during summer than during winter.

To further analyze the performance of the algorithms, numerical results are given in Table IV. Although Selfish
Control gives the worst results (-17\%) and Algorithms 1\&2 give the best results (20\%) in one day simulation, Selfish Control achieves better performance with $0.5 \%$ and Algorithms $\mathbf{1} \& 2$ provide lower benefits with $2.8-3.3 \%$ in the annual simulation. This shows that the saved energy in the battery is used for next day consumption, as mentioned in Section V-A; thus Selfish Control shows a slightly better performance than Baseline in annual simulations. Reasons of the lowered performance for Algorithms 1\&2 include i) not enough PV generation during the winter season, and ii) batteries are not allowed to charge from the main grid.

Secondly, we compare the neighborhood average peak consumption of the algorithms. It can be seen that Selfish and Algorithms 1\&2 lower the peak consumption of the neighborhood more than Baseline. However, the change for Selfish Control is insignificant.

Finally, we define three performance metrics to investigate the success rates (SR) of the algorithms in details:

- SR-01 is the percentage of the smart homes which have reduced their electricity bills.

- $S R-02$ is the percentage of the successful days for which the neighborhood cost has been reduced.

- $S R-03$ is the percentage of the successful days for which

(a)

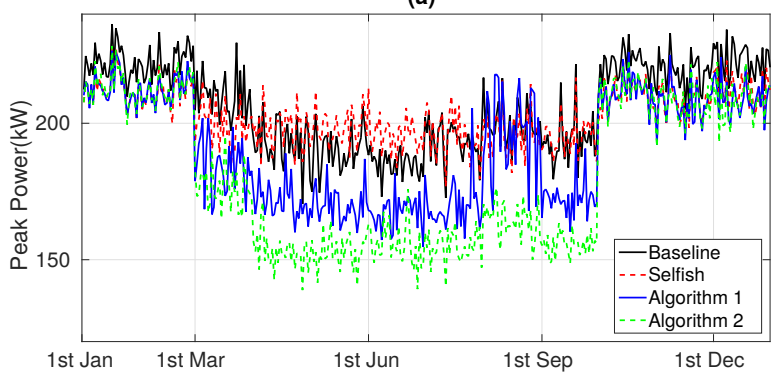

(b)

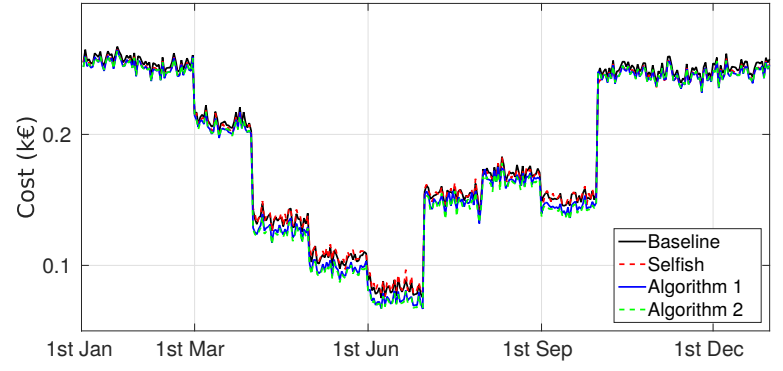

Fig. 5. Annual neighborhood (a) peak consumption, (b) cost profiles of the algorithms without considering forecasting errors. 
TABLE IV

ANNUAL ELECTRIC ENERGY MANAGEMENT WITH (WE) AND WITHOUT (WOE) FORECASTING ERRORS.

\begin{tabular}{|c|c|c|c|c|c|c|c|c|c|c|c|c|c|c|}
\hline & \multicolumn{2}{|c|}{ Cost $(1000 \mathrm{x} €)$} & \multicolumn{2}{|c|}{ Cost $(\%)$} & \multicolumn{2}{|c|}{ Avg. Peak (kW) } & \multicolumn{2}{|c|}{ Peak (\%) } & \multicolumn{2}{|c|}{$S R-01(\%)$} & \multicolumn{2}{|c|}{$S R-02(\%)$} & \multicolumn{2}{|c|}{ SR-03 (\%) } \\
\hline & WoE & WE & WoE & WE & WoE & WE & WoE & WE & WoE & WE & WoE & WE & WoE & WE \\
\hline Baseline & 69.05 & - & - & - & 206.0 & - & - & - & - & - & - & - & - & - \\
\hline Selfish & 68.66 & 68.67 & 0.56 & 0.56 & 202.9 & 203.0 & 1.48 & 1.45 & 78 & 80 & 68.22 & 66.85 & 64.11 & 65.48 \\
\hline Algorithm 1 & 67.07 & 67.09 & 2.86 & 2.84 & 190.6 & 191.0 & 7.48 & 7.25 & 100 & 100 & 99.45 & 98.9 & 95.34 & 94.52 \\
\hline Algorithm 2 & 66.74 & 66.76 & 3.35 & 3.32 & 180.4 & 180.7 & 12.41 & 12.29 & 100 & 100 & 99.73 & 99.73 & 100 & 100 \\
\hline
\end{tabular}

the neighborhood peak consumption has been reduced.

For $S R-01$, it can be seen that all smart homes succeed to decrease their electricity bill with Algorithms 1\&2, while with Selfish Control, around 20 of the smart homes lost money. For home energy management, all smart homes should earn some benefit in return for their efforts. Users may otherwise not be interested in active participation. In this respect, the presented Algorithms 1\&2 show 100\% performance with coordination and energy sharing by battery discharge in the neighborhood. For $S R-02$, Algorithms 1\&2 reduce the electricity cost almost all days $(\sim 360)$, while Selfish reaches $\sim 250$ days. Lastly, for $S R-03$, Algorithm 2 reduces peak consumption all days of the year while Algorithm 1 decreases it for $\sim 350$ days.

Overall, Algorithm 2 gives the best performance in terms of cost and peak reduction, while Algorithm 1 shows slightly lower performance. However, Algorithm 2 requires more computation time (max. $1017 \mathrm{sec}$ ) to coordinate the home agent strategies compared to Algorithm 1 (max. 50 sec.). Up to 100 smart homes, Algorithm 2 can solve the coordination problem in acceptable time limits. However, for larger neighborhoods (such as 1000 smart homes), Algorithm 1 seems preferable. Lastly, Selfish Control is not an effective method for scheduling and battery management in the neighborhood.

\section{DISCUSSION}

This study has demonstrated that coordination with energy sharing provides efficient electric energy management of smart homes, even when considering forecasting errors. However, several simplifying assumptions were made and challenges remain. First, an important constraint is the hypothesis that batteries cannot charge from the grid, hence the cost and peak consumption reductions cannot be generalized. Therefore, as there is less solar radiation in winter, the proposed algorithms have less energy to share in the neighborhood. One can also mention that numerical results $(3.35 \%$ cost and $12.41 \%$ average peak reduction) cannot be generalized as is, as they depend on the market structure, end-users preferences and irradiance profile, but the presented coordination algorithm gives promising results on the annual scale (including for extreme conditions), as shown by the various metrics.

Although the cost savings may seem limited, the potential savings from the PAR reduction should also be considered, e.g., through deferring utility investments. An increase in the penetration rates of $\mathrm{PV}$ and batteries, as well as the integration of electric vehicles with vehicle-to-home technology could further increase these results. Moreover, the direct cost of the coordination would be limited in terms of specific required equipment, hence energy sharing would provide additional benefits with a potentially short payback time. A detailed analysis would however be necessary.

Moreover, smart homes only use the dynamic price calculated by the aggregator during the optimization, hence they cannot anticipate the effect of changing strategies on the price. The reason is that home agents do not know the parameters of the pricing scheme, as we assume that the utility and the aggregator do not want to disclose their profit. As a consequence, this may lead to a high number of iterations. In our case, although the number of iterations never exceeded the maximum limit (20), they tend to be higher on sunny days due to the increased possibilities for energy sharing.

The possible avenues for further exploration of the studied topic also include $i$ ) considering constraints on the distribution grid, such as transformer and line capacity, ii) using empirical data or a dedicated algorithm for forecasting, rather than using simple Gaussian and Gamma distributions, iii) running a sensitivity analysis to explore the impact of different PV and battery sizing values, and $i v$ ) considering the capital costs for PV and battery in a life cycle analysis.

\section{CONCLUSION}

This paper has presented an energy management and sharing strategy to reduce the electricity bills of consumers via coordination of smart homes in a neighborhood. The proposed algorithms aim to utilize neighborhood PV generation efficiently by scheduling the use of resources (appliances, batteries) and sharing energy among neighbors. Two types of coordination methods are presented: group-based and turnbased. Both models show good performance by reducing cost and peak load. Between the coordination methods, for an annual simulation, Algorithm 2 gives the best results with $3.35 \%$ cost and $12.41 \%$ average peak reduction, while Algorithm 1 achieves $2.86 \%$, and $7.48 \%$, compared to a Baseline scenario.

\section{REFERENCES}

[1] A. Zipperer, P. A. Aloise-Young, S. Suryanarayanan, R. Roche, L. Earle, D. Christensen, P. Bauleo, and D. Zimmerle, "Electric energy management in the smart home: Perspectives on enabling technologies and consumer behavior," Proceedings of the IEEE, vol. 101, no. 11, pp. 2397-2408, Nov 2013.

[2] A. A. Khan, S. Razzaq, A. Khan, F. Khursheed, and Owais, "HEMSs and enabled demand response in electricity market: An overview," Renewable and Sustainable Energy Reviews, vol. 42, pp. 773 - 785, 2015. 
[3] S. Althaher, P. Mancarella, and J. Mutale, "Automated demand response from home energy management system under dynamic pricing and power and comfort constraints," IEEE Transactions on Smart Grid, vol. 6, no. 4, pp. 1874-1883, July 2015.

[4] P. Yi, X. Dong, A. Iwayemi, C. Zhou, and S. Li, "Real-time opportunistic scheduling for residential demand response," IEEE Transactions on Smart Grid, vol. 4, no. 1, pp. 227-234, March 2013.

[5] Y. Li, B. L. Ng, M. Trayer, and L. Liu, "Automated residential demand response: Algorithmic implications of pricing models," IEEE Transactions on Smart Grid, vol. 3, no. 4, pp. 1712-1721, Dec 2012.

[6] N. G. Paterakis, I. N. Pappi, J. P. S. Catalo, and O. Erdinc, "Optimal operational and economical coordination strategy for a smart neighborhood," in PowerTech, 2015 IEEE Eindhoven, June 2015, pp. 1-6.

[7] B. Moradzadeh and K. Tomsovic, "Two-stage residential energy management considering network operational constraints," IEEE Transactions on Smart Grid, vol. 4, no. 4, pp. 2339-2346, Dec 2013.

[8] T. M. Hansen, R. Roche, S. Suryanarayanan, A. A. Maciejewski, and H. J. Siegel, "Heuristic optimization for an aggregator-based resource allocation in the smart grid," IEEE Transactions on Smart Grid, vol. 6 , no. 4, pp. 1785-1794, July 2015.

[9] R. Dai and M. Mesbahi, "Optimal power generation and load management for off-grid hybrid power systems with renewable sources via mixed-integer programming," Energy Conversion and Management, vol. 73 , pp. $234-244,2013$.

[10] H. K. Nguyen, J. B. Song, and Z. Han, "Demand side management to reduce peak-to-average ratio using game theory in smart grid," in Computer Communications Workshops (INFOCOM WKSHPS), 2012 IEEE Conference on, March 2012, pp. 91-96.

[11] B. Celik, R. Roche, D. Bouquain, and A. Miraoui, in Cyber-PhysicalSocial Systems and Constructs in Electric Power Engineering, S. Suryanarayanan, R. Roche, and T. M. Hansen, Eds. London: IET, 2016, ch. Increasing renewable local energy use in smart neighborhoods through coordinated trading, pp. 217-252.

[12] A. H. Mohsenian-Rad, V. W. S. Wong, J. Jatskevich, R. Schober, and A. Leon-Garcia, "Autonomous demand-side management based on game-theoretic energy consumption scheduling for the future smart grid," IEEE Transactions on Smart Grid, vol. 1, no. 3, pp. 320-331, Dec 2010

[13] H. Chen, Y. Li, R. H. Y. Louie, and B. Vucetic, "Autonomous demand side management based on energy consumption scheduling and instantaneous load billing: An aggregative game approach," IEEE Transactions on Smart Grid, vol. 5, no. 4, pp. 1744-1754, July 2014.

[14] P. Samadi, H. Mohsenian-Rad, R. Schober, and V. W. S. Wong, "Advanced demand side management for the future smart grid using mechanism design," IEEE Transactions on Smart Grid, vol. 3, no. 3, pp. 1170-1180, Sept 2012.

[15] Y. Guo, M. Pan, Y. Fang, and P. P. Khargonekar, "Decentralized coordination of energy utilization for residential households in the smart grid," IEEE Transactions on Smart Grid, vol. 4, no. 3, pp. 1341-1350, Sept 2013.

[16] S. Kahrobaee, R. A. Rajabzadeh, L. K. Soh, and S. Asgarpoor, "A multiagent modeling and investigation of smart homes with power generation, storage, and trading features," IEEE Transactions on Smart Grid, vol. 4, no. 2, pp. 659-668, June 2013.

[17] B. Celik, R. Roche, D. Bouquain, and A. Miraoui, "Coordinated energy management using agents in neighborhood areas with res and storage," in 2016 IEEE International Energy Conference (ENERGYCON), April 2016, pp. 1-6.

[18] R. Roche, B. Blunier, A. Miraoui, V. Hilaire, and A. Koukam, "Multiagent systems for grid energy management: A short review," in IECON 2010 - 36th Annual Conference on IEEE Industrial Electronics Society, Nov 2010, pp. 3341-3346.

[19] A. Agnetis, G. de Pascale, P. Detti, and A. Vicino, "Load scheduling for household energy consumption optimization," IEEE Transactions on Smart Grid, vol. 4, no. 4, pp. 2364-2373, Dec 2013.

[20] C. Keerthisinghe, G. Verbic, and A. C. Chapman, "A fast technique for smart home management: Adp with temporal difference learning," IEEE Transactions on Smart Grid, vol. PP, no. 99, pp. 1-1, 2016.

[21] N. G. Paterakis, O. Erdin, I. N. Pappi, A. G. Bakirtzis, and J. P. S. Catalo, "Coordinated operation of a neighborhood of smart households comprising electric vehicles, energy storage and distributed generation," IEEE Transactions on Smart Grid, vol. 7, no. 6, pp. 2736-2747, Nov 2016.

[22] M. A. A. Pedrasa, T. D. Spooner, and I. F. MacGill, "Coordinated scheduling of residential distributed energy resources to optimize smart home energy services," IEEE Transactions on Smart Grid, vol. 1, no. 2, pp. 134-143, Sept 2010.
[23] L. Gkatzikis, I. Koutsopoulos, and T. Salonidis, "The role of aggregators in smart grid demand response markets," IEEE Journal on Selected Areas in Communications, vol. 31, no. 7, pp. 1247-1257, July 2013.

[24] S. Shao, M. Pipattanasomporn, and S. Rahman, "Development of physical-based demand response-enabled residential load models," IEEE Transactions on Power Systems, vol. 28, no. 2, pp. 607-614, May 2013.

[25] Z. Tan, P. Yang, and A. Nehorai, "An optimal and distributed demand response strategy with electric vehicles in the smart grid," IEEE Transactions on Smart Grid, vol. 5, no. 2, pp. 861-869, March 2014.

[26] K. Hollands and R. Huget, "A probability density function for the clearness index, with applications," Solar Energy, vol. 30, no. 3, pp. $195-209,1983$

[27] Z. Zhu, S. Lambotharan, W. H. Chin, and Z. Fan, "A game theoretic optimization framework for home demand management incorporating local energy resources," IEEE Transactions on Industrial Informatics, vol. 11, no. 2, pp. 353-362, April 2015.

[28] Z. Chen, L. Wu, and Y. Fu, "Real-time price-based demand response management for residential appliances via stochastic optimization and robust optimization," IEEE Transactions on Smart Grid, vol. 3, no. 4, pp. 1822-1831, Dec 2012.

[29] C. Chen, J. Wang, Y. Heo, and S. Kishore, "Mpc-based appliance scheduling for residential building energy management controller," IEEE Transactions on Smart Grid, vol. 4, no. 3, pp. 1401-1410, Sept 2013.

[30] C. R. Rivero, V. Sauchelli, H. D. Patio, J. A. Pucheta, and S. Laboret, "Long-term power consumption demand prediction: A comparison of energy associated and bayesian modeling approach," in 2015 Latin America Congress on Computational Intelligence (LA-CCI), Oct 2015, pp. 1-6.

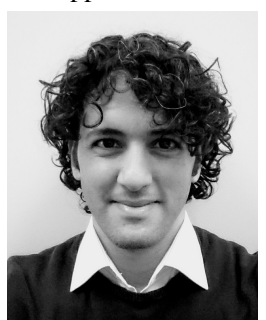

Berk Celik received B.Sc. and M.Sc. degrees from Ege University, Turkey in 2011 and 2013, respectively, both in electrical and electronics engineering. $\mathrm{He}$ is currently pursuing the Ph.D. degree in FEMTO-ST Institute and Energy Department at Université de Technologie de Belfort-Montbéliard, France. His current research interests include power systems, integration of renewable energies, demand response, and the coordination of smart homes.

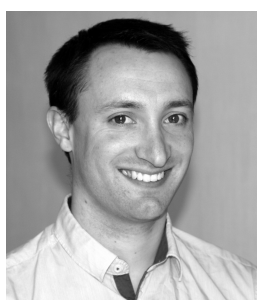

Robin Roche received the Ph.D. degree in electrical engineering from the Université de Technologie de Belfort-Montbéliard (UTBM), France, in 2012. He is currently an Associate Professor at Université Bourgogne Franche-Comté and UTBM, at the FEMTOST Institute, as well as at the FCLAB research center. $\mathrm{He}$ is the chair of the microgrids group at FEMTO-ST. His research interests include microgrids, energy management techniques, as well as renewable energy and storage integration.

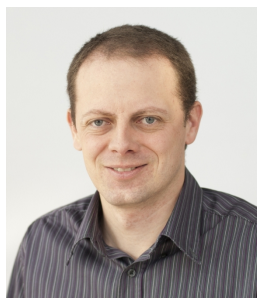

David Bouquain received the M.Sc. degree in Electrical Engineering in 1999 from the Franche-Comté University, France. From 2000 to 2002 he has been engineer at the L2ES Laboratory. He worked on a prototype of hybrid truck for the French army. In 2008, he received the Ph.D. degree in Electrical Engineering from UTBM. Currently he is associate professor within the Energy Department of UTBM. $\mathrm{He}$ is conducting researches at the FEMTO-ST laboratory. His research fields are the design and the energy management of electric vehicles, plug-in hybrid vehicles and fuel cell systems.

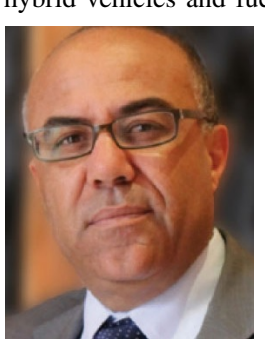

Abdellatif Miraoui (M'07-SM'09) received the M.Sc. degree from Haute Alsace University, France, in 1988, and the Ph.D. degree and the Habilitation to Supervise Research from the University of FrancheComte, France, in 1992 and 1999 respectively. He is the President of Cadi Ayyad University, Morocco. He has been a Full Professor of electrical engineering (electrical machines and energy) at the University of Technology of Belfort-Montbéliard, France, since 2000 . His special interests include fuel cell energy, energy management in transportation, and design and optimization of electrical propulsions/tractions. Prof. Miraoui is the Editor of the International Journal on Electrical Engineering Transportation. $\mathrm{He}$ is a Doctor Honoris Causa of the Technical University of Cluj-Napoca, Romania. He was also distinguished by the University of Brasov, Romania, as an Honorary Professor. 